\title{
Studies on Hyperspectral Face Recognition in Visible Spectrum with Feature Band Selection
}

\author{
Wei Di ${ }^{\text {a }}$, Student Member, IEEE, Lei Zhang ${ }^{b^{*}}$, Member, IEEE \\ David Zhang ${ }^{\mathrm{b}}$, Fellow, IEEE, and Quan Pan ${ }^{\mathrm{c}}$, Member, IEEE \\ ${ }^{a}$ Laboratory for Applications of Remote Sensing, School of Civil Engineering, Purdue University, West \\ Lafayette, IN, USA \\ ${ }^{\mathrm{b}}$ Biometric Research Center, The Hong Kong Polytechnic University, Hong Kong, China \\ ${ }^{\mathrm{c}}$ School of Automation, Northwestern Polytechnical University, Xi'an, China
}

\begin{abstract}
:
This paper studies face recognition by using hyperspectral imagery in the visible light bands. The spectral measurements over the visible spectrum have different discriminatory information for the task of face identification, and it is found that the absorption bands related to hemoglobin are more discriminative than the other bands. Therefore, feature band selection based on the physical absorption characteristics of face skin is performed and two feature band subsets are selected. Then, three methods are proposed for hyperspectral face recognition, including whole band (2D) ${ }^{2} \mathrm{PCA}$, single band (2D) ${ }^{2}$ PCA with decision level fusion, and band subset fusion based (2D) $)^{2} \mathrm{PCA}$. A simple yet efficient decision level fusion strategy is also proposed for the later two methods. To testify the proposed techniques, a hyperspectral face database was established, which contains 25 subjects and has 33 bands over the visible light spectrum $(0.4 \mu \mathrm{m}-0.72 \mu \mathrm{m})$. The experimental results demonstrated that multispectral face recognition with the selected feature bands outperforms that by using a single band, or using the whole bands, or, interestingly, using the conventional RGB color bands.
\end{abstract}

Keywords: Face recognition, hyperspectral imaging, band selection, PCA

\section{Introduction}

Multispectral and hyperspectral imaging can obtain a set of spatially co-registered images at spectrally-contiguous wavelengths, which was first developed for remote sensing purpose ${ }^{[1]}$, and now widely used in agriculture, forest, mineral exploration and environment reconnaissance ${ }^{[1-3]}$. Recently, it has been applied to other areas such as biometrics, skin diagnosis, etc. ${ }^{[4-12]}$. Especially, some studies on hyperspectral face recognition have been reported very recently ${ }^{[8-10[12]}$.

\footnotetext{
* Corresponding author. Email: cslzhang@comp.polyu.edu.hk. This research is supported by the General Research Fund of Hong Kong (PolyU 5351/08E) and the National Natural Science Foundation of China under grant no. 60634030.
} 
Compared with the traditional gray and color facial image data, hyperspectral imaging can acquire the intrinsic spectral information of the skin at many delicate wavelengths, adding to the spatial information, which may reveal the skin information in vivo of reflected, absorbed and emitted electromagnetic energy. It can capture distinctive personal identification patterns determined by their molecular composition that relates to tissue, blood, and structure, etc. Consequently, this leads to the possible application of hyperspectral imaging to face recognition, and has the potential to overcome the difficulties in traditional face recognition, such as the variances of face orientations and expression ${ }^{[8-10][12][31]}$. Pan et al. ${ }^{[8[[]]}$ explored the facial tissue spectral measurements for hyperspectral face recognition in the near-infrared spectral range $(0.7 \mu \mathrm{m}-1.0 \mu \mathrm{m})$. Denes et al. ${ }^{[10]}$ used 3 single visible bands $(0.6 \mu \mathrm{m}, 0.7 \mu \mathrm{m}$, and $0.8 \mu \mathrm{m})$ to test the spectral asymmetry. Chang et al. ${ }^{[11]}$ fused the hyperspectral images in the visible spectrum $(0.4 \mu \mathrm{m}-0.72 \mu \mathrm{m})$ into a single image and compared the result with the visible image to validate the improvement of face recognition due to the image fusion. Chou et al. ${ }^{[12]}$ pre-processed the hyperspectral images (visible: $0.4 \mu \mathrm{m}-0.72 \mu \mathrm{m}$, near-infrared: $0.65 \mu \mathrm{m}-1.1 \mu \mathrm{m})$ by PCA to extract the first principle information for face detection.

All the above studies, however, do not consider the fact that each spectral band represents the specific reflectance/remittance information of the object at that band. Thus, different hyperspectral bands will convey different intrinsic information of human face skin and different discriminatory information for classification. A certain type of tissue might have distinctive spectral reflectance features in some bands, but might not be significant in other bands ${ }^{[13][14]}$. The facial skin has specific spectral characteristics determined by its chemical components ${ }^{[15][16]}$, and their spectral absorption range may convey more information than other bands for face recognition. This paper proposes a feature band selection method by exploiting the intrinsic spectral absorption characteristics of the skin in vivo.

Once the feature bands are selected, the next question is how to process the data for face recognition. Among various face recognition methods ${ }^{[17-21][29-31]}$, the bidirectional $2 \mathrm{DPCA}^{[21]}$ (denoted here as $\left.(2 \mathrm{D})^{2} \mathrm{PCA}\right)$ method, which can be viewed as an extension of the $2 \mathrm{DPCA}^{[20]}$ by simultaneously considering the row and column directions, has the advantage of directly computing the eigenvectors of the image covariance matrix without matrix-to-vector conversion. Therefore, it can alleviate greatly the small sample size (SSS) problem in PCA while requiring a lower computational burden. In this study, we use (2D) ${ }^{2} \mathrm{PCA}$ as the basic technique for face recognition.

Then, with different considerations, three types of methods are proposed. First, it is natural to treat each feature band as different patterns and use them all as the input to $(2 \mathrm{D})^{2} \mathrm{PCA}$ for recognition. The second way is to process each band separately and to do the decision level fusion to obtain the final recognition result. Third, some subsets of feature bands 
can be constructed and fused within each subset due to the high correlation between neighbouring bands. Each fused image can be used separately to obtain its own (2D) ${ }^{2}$ PCA projection matrix. Feature band fusion can help to remove the redundancies between neighbouring bands and the noise, which also efficiently combines different characteristics of images $^{[22][23]}$. Here, we focus on the weighted average fusion ${ }^{[22]}$ and Laplacian Pyramid image fusion ${ }^{[23]}$. The recognition results of each subset are then fused at the decision level, whereby a Distance and Voting rule is proposed.

The experiments are based on a newly built database using a novel hyperspectral face acquisition system. Two key feature bands are selected (540nm and 580nm) and three methods are proposed: whole band based (2D) ${ }^{2} \mathrm{PCA}$ (WB for short), single band based (2D) ${ }^{2} \mathrm{PCA}$ with decision level fusion (SBD for short), and band subset fusion based (2D) ${ }^{2} \mathrm{PCA}$ with different image fusion methods (BS-xFD for short). Results demonstrated the effectiveness of our proposed band selection method, which outperforms methods using either a single band or using the whole bands. Moreover, by simulating the RGB channels, we demonstrated that the selected absorption feature bands perform better than the conventional RGB bands in face recognition. This work implies that a new multispectral face recognition system based on the selected feature bands could be developed in the near feature.

\section{Database Establishment}

Researchers have recently studied the feasibility of hyperspectral face image recognition. The Munsell Color Science Laboratory established the Lippmann2000 database ${ }^{[28]}$ which contains hyperspectral images from 4 Caucasians and 3 East-Asians. Pan et al. ${ }^{[8[9]}$ acquired spectral images over the near-infrared spectrum $(0.7-1 \mu \mathrm{m})$ from 200 human participants and showed the effectiveness of near-infrared hyperspectral image for face recognition under different postures and expressions. Chang et al. ${ }^{[1]}$ established a database that contains 82 participants of different ethnicities, ages, facial hair characteristics and genders over the visible spectrum under different illumination: halogen light, fluorescent light and day light. The Carnegie Mellon University constructed a database of 18 subjects with 54 diverse faces at multiple sessions over a two-month period to test spectral asymmetry for personal identification ${ }^{[10]}$.

However, none of the above database is publicly available. In our study, we built an indoor hyperspectral face acquisition system which mainly consists of a CRI's VariSpec LCTF and a Halogen Light (Illustrated in Fig. 1), and collected a hyperspectral dataset of 300 hyperspectral image cubes from 25 volunteers with age range from 21 to 33 (8 female and 17 male). For each individual, 4 sessions were collected at two different times ( 2 sessions each time) with an average time span of 5 months. The minimal interval is 3 months and the maximum is 10 months. Each session consists 
of three hyperspectral cubes - frontal, right and left views with neutral-expression. The spectral range is from 400nm to $720 \mathrm{~nm}$ with a step length of $10 \mathrm{~nm}$, producing 33 bands in all. Fig. 2 shows an example of the hyperspectral face. Since the database was constructed over a long period of time, significant appearance variations of the subjects, e.g. changes of hair style and skin condition, are presented in the data. In data collection, positions of the camera, light and subject are fixed, which allows us to concentrate on the spectral characteristics for face recognition without masking from environmental changes.

\section{Feature Band Selection}

The facial skin color is determined by its chemical components that have specific spectral characteristics ${ }^{[15][16]}$ and may contribute differently for face recognition. Thus, a feature band selection method that based on the intrinsic biomedical absorption characteristics of the skin in vivo is proposed aiming to preserve the most informative bands while removing bands that convey little discriminatory information.

The face skin color is affected by many factors, such as blood flow, water concentration blood oxygenation, melanin concentration, even aging, makeup or the perspiration level ${ }^{[15][29]}$. Generally, skin color is most affected by three types of light-absorbing chemical compounds: carotene, melanin and haemoglobin ${ }^{[15]}$. However, compared with haemoglobin, the amount of carotene is very little, and melanin is more likely influenced by the outside factors such as sunlight ${ }^{[15] 16]}$. Fig. 3 illustrates the absorption characteristics of hemoglobin and melanin ${ }^{[15]}$. Fig. 4 shows the calibrated skin reflectance ( $2 \mathrm{~nm}$ bandwidth) of 4 individuals under two lighting conditions from our dataset. It clearly shows $\quad$ two hemoglobin absorption bands (around 540nm and 580nm) which correspond to Fig. 3. Thus, in our study two feature band subsets centred at the peak absorption bands are selected in order to better describe the absorption valley (one consists of bands at $530 \mathrm{~nm}, 540 \mathrm{~nm}$ and $550 \mathrm{~nm}$, the other one contains the bands at 570nm, $580 \mathrm{~nm}$ and $590 \mathrm{~nm}$ ). The hemoglobin absorption peak at $420 \mathrm{~nm}$ is not considered due to the low signal-to-noise-ratio at this band in our system. In the following, we propose three classes of hyperspectral face recognition algorithm in the context of $(2 \mathrm{D})^{2} \mathrm{PCA}$, by which the experimental results validate that the selected absorption feature bands are indeed the most informative and lead to higher recognition rate than using any single band or using the entire bands.

\section{Algorithms}

Suppose that we have $S$ hyperspectral data cubes for each of the $M$ subjects. Denote by $N$ the number of feature band 
subsets. For the $i^{\text {th }}$ feature band subset, there are $I_{i}$ bands. Then we denote by $\mathbf{X}_{m s i}^{b}\left(1 \leq b \leq I_{i}, 1 \leq i \leq N\right)$ the $b^{\text {th }}$ band image of the $i^{\text {th }}$ feature band subset for the $m^{\text {th }}$ individual in the $s^{\text {th }}$ cube. Here, we employ (2D) $)^{2}$ PCA as the base method and 3 algorithms are proposed: whole band (2D) ${ }^{2} \mathrm{PCA}$, single band (2D) ${ }^{2} \mathrm{PCA}$ with decision level fusion, and band subset fusion based (2D) ${ }^{2} \mathrm{PCA}$ with decision fusion.

\subsection{Whole Band (2D) ${ }^{2}$ PCA}

An intuitive idea to process the hyperspectral data is to view all the selected hyperspectral bands as different patterns at the corresponding wavelength, and process them by using the standard (2D) ${ }^{2} \mathrm{PCA}$ method. We refer to this method as the Whole Band (2D) ${ }^{2} \mathrm{PCA}$ (abbreviated by WB here). The covariance matrices along the row and column directions in the image space are computed as:

$$
\begin{aligned}
& \mathbf{G}_{1}=\frac{1}{M S\left(\sum_{i=1}^{N} I_{i}\right)} \sum_{m=1}^{M} \sum_{s=1}^{S} \sum_{i=1}^{N} \sum_{b=1}^{I_{i}}\left(\mathbf{X}_{m s i}^{b}-\overline{\mathbf{X}}\right)^{T}\left(\mathbf{X}_{m s i}^{b}-\overline{\mathbf{X}}\right) \\
& \mathbf{G}_{2}=\frac{1}{M S\left(\sum_{i=1}^{N} I_{i}\right)} \sum_{m=1}^{M} \sum_{s=1}^{S} \sum_{i=1}^{N} \sum_{b=1}^{I_{i}}\left(\mathbf{X}_{m s i}^{b}-\overline{\mathbf{X}}\right)\left(\mathbf{X}_{m s i}^{b}-\overline{\mathbf{X}}\right)^{T}
\end{aligned}
$$

where $\overline{\mathbf{X}}=\frac{1}{M S\left(\sum_{i=1}^{N} I_{i}\right)} \sum_{m=1}^{M} \sum_{s=1}^{S} \sum_{i=1}^{N} \sum_{b=1}^{I_{i}} \mathbf{X}_{m s i}^{b}$. The projection matrix $\mathbf{V}_{1}=\left[\mathbf{v}_{11}, \mathbf{v}_{12}, \ldots, \mathbf{v}_{1 k_{1}}\right]$ for WB is composed of the orthogonal eigenvectors of $\mathbf{G}_{1}$ corresponding to the $k_{1}$ largest eigenvalues, and the projection matrix $\mathbf{V}_{2}=\left[\mathbf{v}_{21}, \mathbf{v}_{22}, \ldots, \mathbf{v}_{2 k_{2}}\right]$ consists of the orthogonal eigenvectors of $\mathbf{G}_{2}$ corresponding to the largest $k_{2}$ eigenvalues. $k_{1}$ and $k_{2}$ can be determined by setting a threshold to the cumulant eigenvalues $\mathrm{Cu}$.

Similarly, for each test hyperspectral data cube, we first extract its feature band subsets and then project each band image, denoted by $\widetilde{\mathbf{X}}_{i}^{b}\left(1 \leq b \leq I_{i}, 1 \leq i \leq N\right)$, by $\mathbf{V}_{1}$ and $\mathbf{V}_{2}$ to the projection space. The distance of the test data to the $m^{\text {th }}$ individual is defined as:

$$
d_{m}=\frac{1}{\sum_{i=1}^{N} I_{i}} \sum_{i=1}^{N} \sum_{b=1}^{I_{i}}\left\|\mathbf{V}_{2}^{T} \tilde{\mathbf{X}}_{i}^{b} \mathbf{V}_{1}-\mathbf{Y}_{m}\right\|
$$

where $\mathbf{Y}_{m}=\frac{1}{S\left(\sum_{i=1}^{N} I_{i}\right)} \sum_{s=1}^{S} \sum_{i=1}^{N} \sum_{b=1}^{I_{i}} \mathbf{V}_{2}^{T} \mathbf{X}_{m s i}^{b} \mathbf{V}_{1}$ is the average of all training data of the $m^{\text {th }}$ individual in the projection space. The final classification of the test hyperspectral data is made as the one that gives the minimum distance:

$$
\hat{j}=\underset{m}{\arg \min } d_{m}
$$




\subsection{Single Band (2D) ${ }^{2}$ PCA and Feature Band Subset (2D)²PCA}

The above proposed WB method takes all the selected bands equally as the input of (2D) ${ }^{2} \mathrm{PCA}$. However, different bands may have quite different characteristics. Due to the limitations of the eigenvalue based method, which uses the variation to measure the importance of patterns, the characteristics of different bands and different identities may be mixed up. Also, since nearby bands tends to have more similar characteristic, by grouping them into separate feature set can further benefit the performance of (2D) $)^{2}$ PCA. Denote by $\left\{\mathbf{X}_{1}, \mathbf{X}_{2}, \ldots, \mathbf{X}_{I_{i}}\right\}^{i}$ the $i^{\text {th }}$ feature sub-band set. For each feature set, by applying formula (1)-(4), a multiple decision set $\left\{\hat{j}_{1}, \hat{j}_{2}, \ldots \hat{j}_{N}\right\}$ can be obtained. The final classification result can be obtained by decision level fusion which will be proposed in section 4.3. Further, if we simply treat each single band as a sub-band set, then $N$ pairs of projection matrix can be obtained, and the recognition performance of each single band can be obtained by the projection matrix that corresponds to the same single band obtained from training. In the following, this Single Band (2D)²PCA method is called SB for short.

Furthermore, since different bands in hyperspectral imagery contain complementary information, additional improvements may be obtained by fusing bands in each feature sub-band set into a single image. The pixel-level image fusion could extract, pixel-by-pixel, the most important information from each spectral band and result in a single fused image without much distortion or loss of information. A general formula of image fusion is:

$$
\mathbf{F}^{i}=f\left(\left\{\mathbf{X}_{1}, \mathbf{X}_{2}, \ldots, \mathbf{X}_{I_{i}}\right\}^{i}\right)
$$

where $\mathbf{F}^{i}$ is the fused image obtained from the $i^{\text {th }}$ feature sub-band set, and $f(\cdot)$ represents the fusion function. Two pixel-level image fusion methods ${ }^{[22][23]}$ are used here. One is the simple weighted averaging: $F^{i}=\sum_{b=1}^{I_{i}} w_{b i} X_{b i}$, where $w_{b i}$ is the weight for the $b^{\text {th }}$ band in the $i^{\text {th }}$ feature band-subset. The other one is Laplacian Pyramid fusion ${ }^{[23]}$, where measurement for coefficient selection is defined as the local energy within a neighbourhood region.

Since each $\mathbf{F}^{i}$ is obtained from a band subset that is non-overlap with others, the difference between them might be significant. Thus each $\mathbf{F}^{i}$ is processed separately using formula (1)-(4), where totally $N$ pairs of projection matrix are constructed, and again, $N$ decision values $\left\{\hat{j}_{1}, \hat{j}_{2}, \ldots \hat{j}_{N}\right\}$ are obtained. To integrate these decisions reasonably, we propose a two step decision making rule, namely the Dv rule, in the next sub-section.

\subsection{Decision Rule by Distance \& Voting (Dv)}


Here we propose a simple decision fusion method based on the majority voting strategy which is proved to be simple but as effective as the other more complicated schemes, such as Bayesian and Dempster-Shafer theory ${ }^{[22][2427]}$.

Denote by $\|\bullet\|$ the cardinality of a set, and by $\Omega_{j}$ the set that consists of the minimum distance $d_{j}^{i}=\min \left(d_{m}^{i}\right), m=1,2, \ldots, M$. The cardinality of $\Omega_{j}$ represents the total number of votes given by each feature band subset that classifies the test data to the $j^{\text {th }}$ class. The two step decision making rule (Dv) is as follows:

Step I. If a unique maximum vote exists, i.e. $\left\|\Omega_{q}\right\|=\max \left\|\Omega_{j}\right\|, j=1,2, \ldots, M$, the final decision is made as:

$$
q=\underset{j}{\arg \max }\left\|\Omega_{j}\right\|
$$

Step II. If $q$ is not unique that satisfies $\left\|\Omega_{q}\right\|=\max \left\|\Omega_{j}\right\|$, then suppose that there are $K$ sets $\Omega_{q_{1}}, \Omega_{q_{2}}, \cdots, \Omega_{q_{K}}$ that satisfy $\left\|\Omega_{q_{1}}\right\|=\left\|\Omega_{q_{2}}\right\|=\cdots=\left\|\Omega_{q_{K}}\right\|=\max \left\|\Omega_{j}\right\|(j=1,2, \ldots, M)$, where $q_{1}, q_{2}, \ldots, q_{K}$, are the $K$ different decisions. We calculate the sum of the distances for each of these $K$ sets $\Omega_{q_{1}}, \Omega_{q_{2}}, \cdots, \Omega_{q_{K}}$ :

$$
S_{q_{k}}^{d}=\sum_{i=1}^{\left\|\Omega_{q_{k}}\right\|} D_{i}, \quad D_{i} \in \Omega_{q_{k}}, 1 \leq k \leq K
$$

where $D_{i}$ is the element of $\Omega_{q_{k}}, 1 \leq i \leq\left\|\Omega_{q_{k}}\right\|$. Then the final decision is made as:

$$
q=\underset{k}{\arg \min } S_{q_{k}}^{d}, 1 \leq k \leq K
$$

In this paper, the proposed Band Subset Fusion Decision scheme using the weighted average image fusion is denoted as BS-WFD, and the one using Laplacian Pyramid fusion with salient measurement selection rule is denoted as BS-LFD.

\section{Experiments}

In the experiment, frontal hyperspectral images of the 25 individuals (each with 4 cubes) were used. The established dataset is available at http://www.comp.polyu.edu.hk/ cslzhang/datasets.htm. Each time, 2 of the 4 cubes were selected to form the training data set, and the left 2 cubes were for testing, leading to 6 different combinations. Average performance was used to evaluate different algorithms. Since all data were acquired under controlled and constant illumination, the radiance data were used directly without calibration. The first 6 and last 3 bands were removed due to high noise, leaving 24 spectral bands in all. The eye coordinates were located manually for image registration, and a sub-region of face with size $162 \times 150$ was cropped from each band, normalized and scaled to a size of $54 \times 50$ image to save the computational cost. A list of all tested algorithms with different settings is given in Table 1. 


\subsection{Experiment 1}

To analyze the performance of different spectral bands for face recognition, we test the recognition rates of each single band by (2D) ${ }^{2} \mathrm{PCA}$ with different cumulant eigenvalue $\mathrm{Cu}$. Projection matrices are constructed for each band and applied to the corresponding band of the test hyperspectral cube which leads to a classification decision of each band. Fig. 5 and Table 2 show the recognition rates of $\mathrm{MB}, \mathrm{WB}(1,24)$ (denoted as $\mathrm{WB})$ and $\mathrm{SBD}(24,1)$ (denoted as $\mathrm{SBD}$ ) under different values of $\mathrm{Cu}$, where "MB" represents the average recognition rate of all bands. The methods $\mathrm{WB}(1,24)$ and $\operatorname{SBD}(24,1)$ are the integration of all the bands, thus they reach moderate recognition results. It is also observed that the recognition rate is not very sensitive to different values of $\mathrm{Cu}$.

It should be noted that the hemoglobin absorption bands (especially at 540nm and 580nm) show much higher recognition rate than other bands. This validates our consideration of feature band selection. Next, we compare the recognition rates of each selected feature band and methods $\operatorname{WB}(2,3,3)$ and $\operatorname{SBD}(2,3,3)$. The results are in Table 3 and plotted in Fig. 6, where MB(6) stands for the average recognition rate of all the six selected feature bands. WB is short for $\mathrm{WB}(2,3,3)$ and $\operatorname{SBD}$ is short for $\operatorname{SBD}(2,3,3)$. It is seen that methods $\operatorname{WB}(2,3,3)$ and $\operatorname{SBD}(2,3,3)$, which use all the selected feature bands, outperform each single feature band. $\operatorname{SBD}(2,3,3)$ achieves slightly higher recognition rate than $\mathrm{WB}(2,3,3)$ since it calculates the projection matrix for each band thus incorporates possible differences between bands.

Comparing the results listed in Table 2 and Table 3, one can notice that by applying the same strategy, such as WB and $\mathrm{SBD}$, methods using the selected feature bands (i.e. $\mathrm{WB}(2,3,3)$ and $\operatorname{SBD}(2,3,3))$ outperform the methods using all 24 bands (i.e. $\mathrm{WB}(1,24)$ and $\operatorname{SBD}(24,1))$. Also, since $\mathrm{SBD}$ separates the band factor from the factor relating to individual subject, it yields a better projection and better performance than WB .

\subsection{Experiment 2}

To test the efficiency of our proposed BS-WFD and BS-LFD algorithms, we applied them to the selected feature bands with a fixed $\mathrm{Cu}=98.5 \%$. Fig. 7 compares the results of the following methods: BS-WFD with 12 weighting cases; BS-LFD; MB(24) by averaging the recognition rates of all the 24 spectral bands and $\mathrm{MB}(6)$ by averaging the recognition rates of all the 6 feature bands. Table 4 lists the recognition rates by 12 different weighting schemes for image fusion. A format of w1-w2-w3 was used to record the weights, e.g. "3-10-1" means that weights ratio for the 3 feature bands in a subset are 3, 10, and 1 respectively with sum-to-one. The central bands (540nm and 580nm) are assigned with higher weights to emphasize their importance. 
From Fig. 7 and Table 4, we can see that the proposed methods BS-WFD and BS-LFD achieve the best performance. They outperform $\mathrm{MB}(6), \mathrm{MB}(24)$ and any single band (the best performance obtained by single band is at 540nm). In comparison with Experiment 1 (Table 3 and Fig. 6), it can also be seen that BS-WFD and BS-LFD are better than SBD and WB. This is because they take advantage of image fusion which may reduce the image noise. The results also show that different weights in BS-WFD do not have significant influence on the recognition rate. Since BS-WFD utilizes the prior information to assign the key absorption bands with higher weights, its performance is better than BS-LFD whereby fusion only depends on information obtained from the image.

\subsection{Experiment 3}

In order to demonstrate the advantage by selecting specific spectral bands for face recognition over the conventional RGB color bands, we simulated RGB images from the red, green and blue bands (centered at $600 \mathrm{~nm}, 560 \mathrm{~nm}$ and 490nm respectively) from the acquired hyperspectral data. Fig. 8 shows some simulated RGB face images. A form of "r-g-b" is used to record how the color image is simulated. For example, "3-5-7" means that 3 bands centered at 600nm, 5 bands centered at $560 \mathrm{~nm}$ and 7 bands centered at $490 \mathrm{~nm}$ are averaged to represent the red, green and blue channel respectively. Balanced and unbalanced color compositions are both shown in Fig. 8. Fig. 9 illustrates the face recognition rate of the simulated color images with balanced color composition.

In Fig. 9, we tested four schemes. The first 3 schemes use only the red bands, green bands or blue bands to simulate the R, G or B color channels by average fusion, and then use standard (2D) ${ }^{2} \mathrm{PCA}$ for face recognition. The red line in Fig. 9 shows the recognition results using only the simulated R channel. Similarly, the green and blue lines show the results of green and red channels respectively. The fourth scheme is to apply the proposed Dv rule to the results obtained by the three simulated RGB channels. It shows that the performance is improved compared with any single R, G or B channel. However, the recognition rate is even much lower than that by using the whole bands $\mathrm{WB}(1,24)$. Also we noted that in some RGB simulation cases, the number of the used bands is even larger than that in the proposed band-selection method, which demonstrates that the selected feature bands which do not strictly locate in the RGB region may contain more useful information for face recognition.

\section{Conclusions}

This paper studied the application of hyperspectral imagery in face recognition. A hyperspectral imaging system was built 
and a hyperspectral face database was established to test the face recognition methods. Considering that the absorption bands of facial skin could highlight the intrinsic biomedical characteristics of the skin in vivo, we conducted feature band selection and proposed three classes of methods for hyperspectral face recognition: WB, SBD and BS-xFD (BS-WFD, BS-LFD). Based on the experimental results, we can make the following conclusions. First, face recognition with feature band selection outperforms that by using either a single band or using the whole bands. Second, BS-xFD takes the advantage of image fusion which may reduce the image noise, thus leads to a better performance than WB and SBD. Also since SBD considers the differences between bands, it outperforms WB. Third, we demonstrated that the selected absorption feature bands perform better than the conventional RGB color bands in face recognition. This implies that a new multispectral face recognition system based on the selected feature bands could be developed in the near feature.

\section{References}

[1] A. Goetz, G. Vane, J. Solomon, B. Rock, "Imaging spectrometry for earth remote sensing”, Science, vol.228, no. 4704, pp.1147-1153, Jun. 1985.

[2] D. Landgrebe, "Hyperspectral image data analysis", IEEE Signal Processing Magazine, vol.19, no.1, pp. 17-28, Jan. 2002.

[3] G. Shaw, D. Manolakis, "Signal processing for hyperspectral image exploitation", IEEE Signal Processing Magazine, vol.19, no.1, pp. 12-16, Jan. 2002.

[4] R. Rowe, K. Nixon, "Fingerprint enhancement using a multispectral sensor", in Proceedings of SPIE Biometric Technology for Human Identification II, Bellingham, WA, vol. 5779, pp. 81-93, Apr. 2005.

[5] P. Mehl, Y. Chen, M. Kim, D. Chan, "Development of hyperspectral imaging technique for the detection of apple surface defects and contaminations", Journal of Food Engineering, vol.61, no.1, pp. 67-81, Jan. 2004.

[6] L. Randeberg, I. Baarstad, T. Loke, P. Kaspersen, "Hyperspectral imaging of bruised skin", in Proceedings of SPIE of Photonic Therapeutics and Diagnostics II, vol.6078, pp. 1-11, Feb. 2006.

[7] I. Kim, M. Kim, Y. Chen, and S. Kong, "Detection of skin tumors on chicken carcasses using hyperspectral fluorescence imaging", Transactions of ASAE, vol.47, no.5, pp. 1785-1792, Oct. 2004.

[8] Z. Pan, G. Healey, M. Prasad, and B. Tromberg, "Face recognition in hyperspectral images", IEEE Trans. Pattern Anal. Mach. Intell. vol.25, no.12, pp. 1552-1560, Dec. 2003.

[9] Z. Pan, G. Healey, M. Prasad, B. Tromberg, "Illumination-invariant face recognition in hyperspectral images", in Proceedings of SPIE: Algorithms and Technologies for Multispectral, Hyperspectral, and Ultraspectral Imagery IX, vol.5093, pp. 275-282, Apr. 2003.

[10] L. Denes, P. Metes, and Y. Liu, "Hyperspectral face database", in Technical Report CMU-RI-TR-02-25, 2002.

[11] H. Chang, H. Harishwaran, M. Yi, A. Koschan, B. Abidi, and M. Abidi, "An indoor and outdoor, multimodal, multispectral and multi-illuminant database for face recognition", in Proc. of CVPR2006, workshop on multi-model biometrics, Jun. 2006.

[12] Y. Chou and P. Bajcsy, "Toward face detection, pose estimation and human recognition from hyperspectral imagery", in Technical Report NCSA-ALG-04-0005, 2004, http://isda.ncsa.uiuc.edu/peter/ [online]

[13] B. Guo, S. Gunn, R. Damper, and J. Nelson, "Band selection for hyperspectral image classification using mutual information", IEEE Geoscience and Remote Sensing Letters, vol.3, no. 4, pp. 522-526, Oct. 2006.

[14] R. Huang, M. He, "Band selection based on feature weighting for classification of hyperspectral data", IEEE Geoscience and Remote Sensing Letters, vol.2, no. 2, pp. 156-159, Apr. 2005.

[15] T. Igarashi, K. Nishino and S. Nayar, "The appearance of human skin", in Technical Report CUCS-02405 Columbia University, 2005.

[16] E. Claridge, S. Cotton, P. Hall, and M. Moncrieff, "From colour to tissue histology - physics based interpretation of images of pigmented skin lesions", Medical Image Analysis, vol.7, Issue 4, pp.489-502, Dec. 2003.

[17] J. Yang, D. Zhang, A. Frangi, and J. Yang, "Two-dimensional PCA: a new approach to appearance-based face representation and recognition", IEEE Trans. Pattern Anal. Mach. Intell. vol.26, no. 1, pp. 131-137, Jan. 2004.

[18] J. Ruiz-del-Solar and P. Navarrete, "Eigenspace-based face recognition: a comparative study of different approaches", IEEE Transactions on Systems, Man and Cybernetics, Part C, vol.35, no. 3, pp. 315-325, Aug. 2005.

[19] J. Yang, D. Zhang, Y. Xu. and J.-y. Yang, “Two-dimensional discriminant transform for face recognition”, Pattern Recognition, vol. 38, no.7, pp. 1125-1129, Jul. 2005.

[20] W. Zuo, D. Zhang, and K. Wang, "Bidirectional PCA with assembled matrix distance metric for image recognition", IEEE Transactions on Systems, Man, and Cybernetics - Part B: Cybernetics, vol.36, no.4, pp. 863-872, Aug. 2006. 
[21] D. Zhang and Z. Zhou, "(2D)²PCA: 2-directional 2-dimensional PCA for efficient face representation and recognition", Neurocomputing, vol.69, Issues 1-3, pp. 224-231, 2005.

[22] R Sims, M Phillips, "Target signature consistency of image data fusion alternatives", Optical Engineering, vol.36, no. 3, pp. 743-754, Mar. 1997.

[23] P. Burt and E. Adelson, "The laplacian pyramid as a compact image code", IEEE Transactions on Communications, vol.31, no.4, pp: 532-540, Apr. 1983.

[24] L. Lam and C. Suen, "Application of majority voting to pattern recognition: an analysis of its behavior and performance", IEEE Transactions on Systems, Man, and Cybernetics - Part A: Systems and Humans, vol.27, no. 5, pp. 553-568, Sept. 1997.

[25] B. Achermann and H. Bunke, "Combination of face classifiers for person identification", in Proc. of Int. Conf. Pattern Recognition, vol 3, pp. 416-420, Aug. 1996.

[26] J. Czyz, J. Kittler, and L. Vandendorpe, "Multiple classifier combination for face-based identity verification", Pattern Recognition,vol. 37, Issue 7, pp. 1459-1469, Jul. 2004.

[27] J. Kittler, M. Hatef, R. Duin, and J. Matas, “On combining classifiers”, IEEE Trans.Pattern Anal. Mach. Intell., vol.20, no. 3, pp. 226-239, Mar. 1998 .

[28] M. Rosen and X. Jiang, "Lippmann2000: A spectral image database under construction", in Proc. of the International Symposium on Multispectral Imaging and Color Reproduction for Digital Archives, pp. 117-122, Oct. 1999.

[29] P. Hallinan, G. Gordon, A. Yuille, P. Giblin, and D. Mumford, "Two - and-three dimensional patterns of the face", A K Peters, Natick, Massachusetts, 1998.

[30] G. Medioni, J. Choi, C. Kuo, and D. Fidaleo, "Identifying noncooperative subjects at a distance using face images and inferred three-dimensional face models", IEEE Trans. Syst., Man, Cybern. A: Syst. Humans, vol.39, no.1, pp.12-24, Jan. 2009

[31] Y. Gao, M. Leung, S. Hui, and M. Tananda, "Facial expression recognition from line-based caricatures," IEEE Trans. Syst., Man, Cybern. A: Syst. Humans, vol. 33, no. 3, pp. 407-412, May 2003. 


\section{List of Figures}

Fig. 1 The established hyperspectral face imaging system.

Fig. 2 Illustration of a set of 33 hyperspectral face bands.

Fig. 3 Absorption characteristics of hemoglobin and melanin

Fig. 4 Skin reflection of 4 individuals under two illumination conditions acquired by our sensing system whereby (a) is with higher light intensity than (b).

Fig. 5 Recognition rate of each single band by (2D) ${ }^{2} \mathrm{PCA}$ and by methods of $\mathrm{WB}(1,24)$ and $\operatorname{SBD}(24,1)$.

Fig. 6 Recognition rate of each selected single feature band and by methods of $\mathrm{WB}(2,3,3)$ and $\operatorname{SBD}(2,3,3)$.

Fig. 7 Recognition rate of each subset by $(2 \mathrm{D})^{2} \mathrm{PCA}$, the proposed methods BS-WFD with 12 weighting cases (denoted from 1 to 12) and BS-LFD.

Fig. 8 Examples of RGB color image simulation.

Fig. 9 Face recognition rate of simulated color images with balanced color composition. 


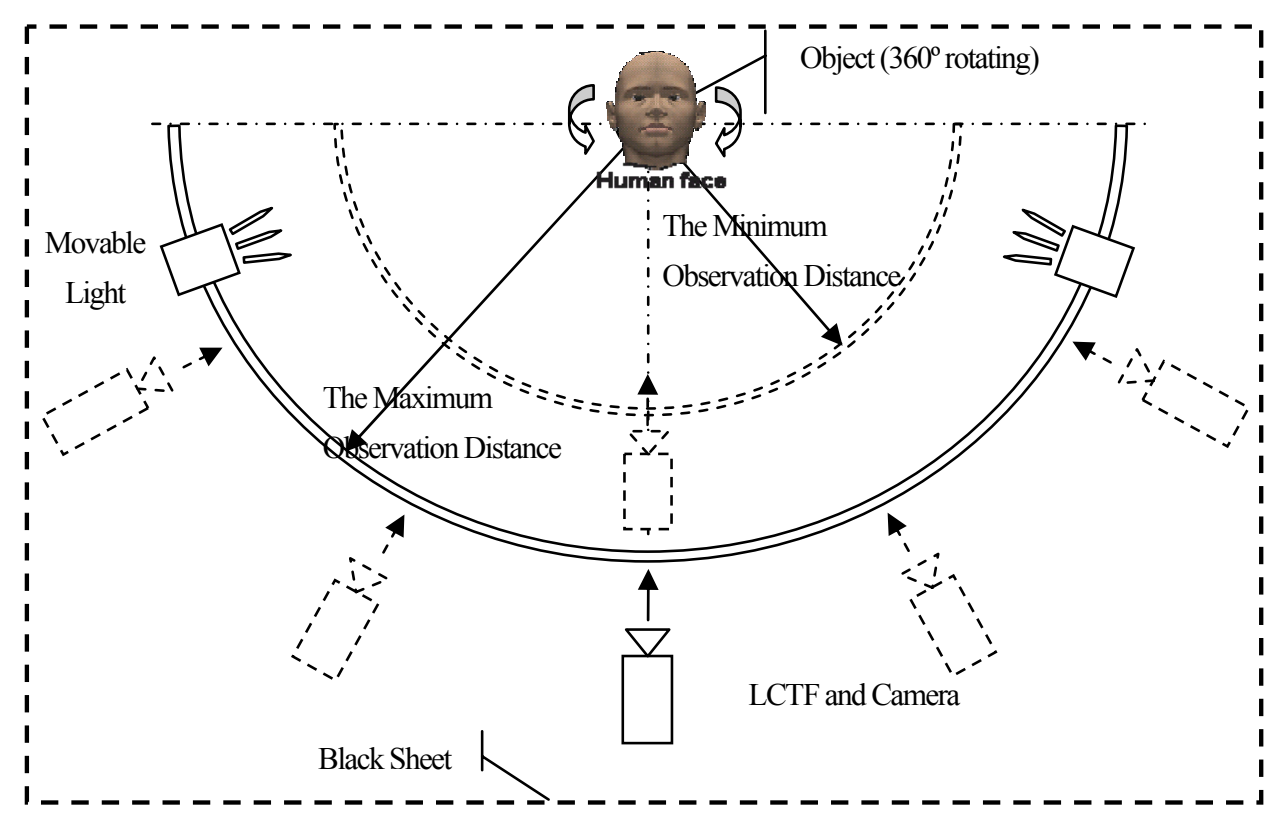

Fig. 1 The established hyperspectral face imaging system. 

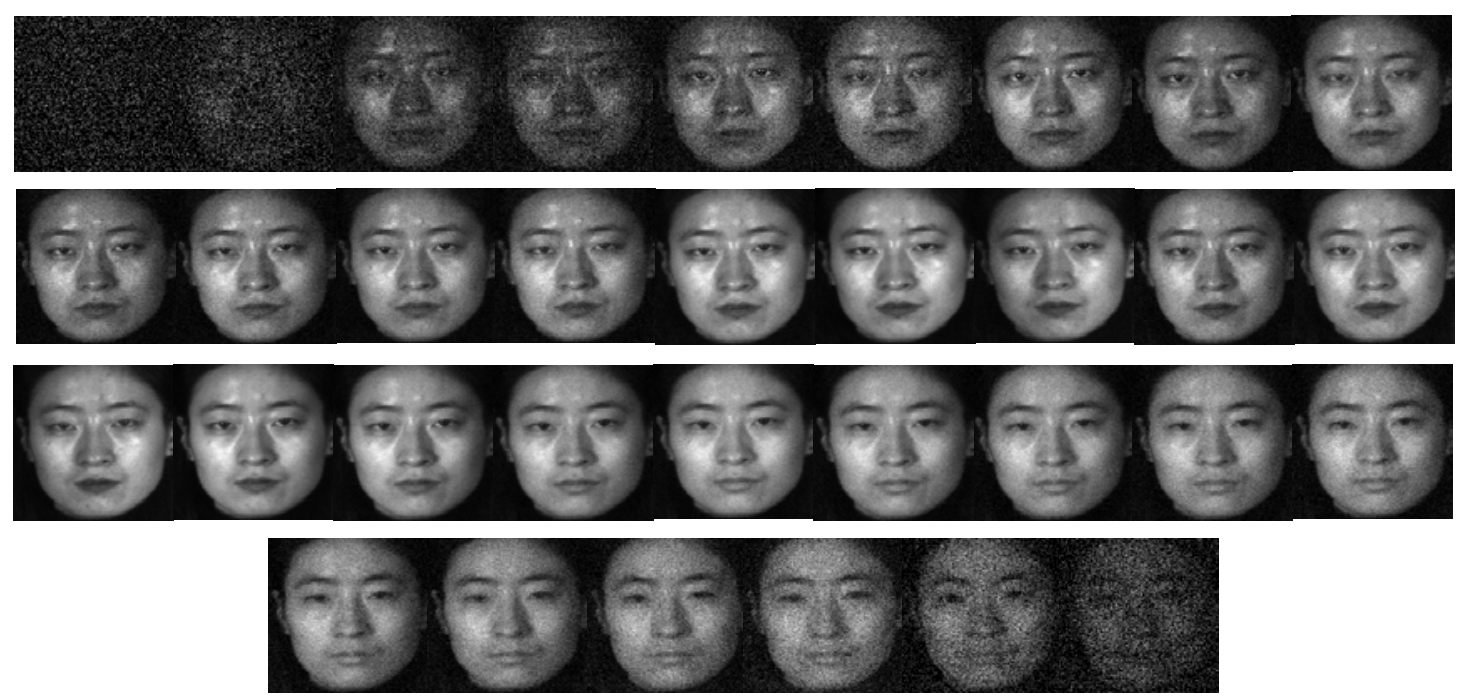

Fig. 2 Illustration of a set of 33 hyperspectral face bands. 


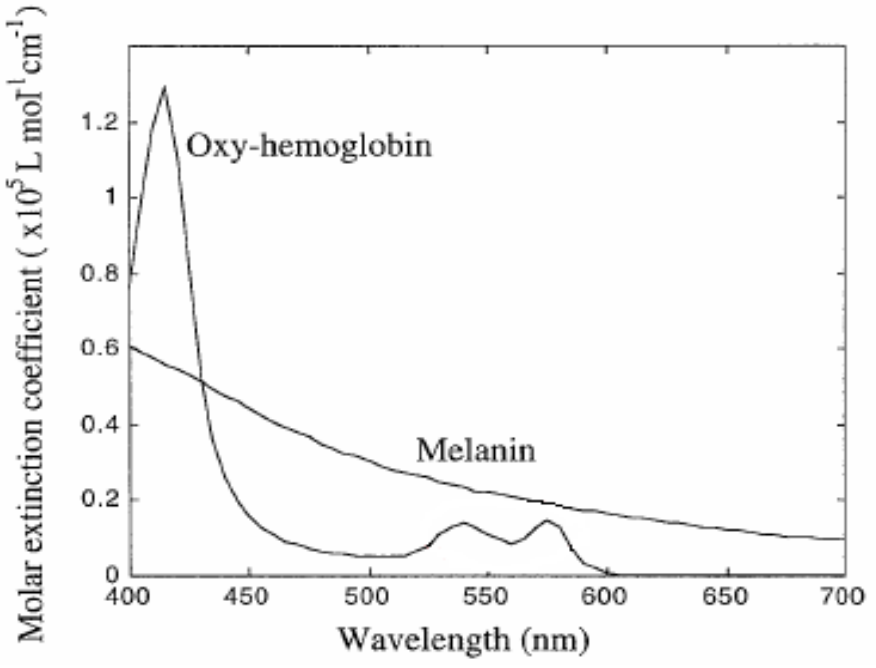

Fig. 3 Absorption characteristics of hemoglobin and melanin. 


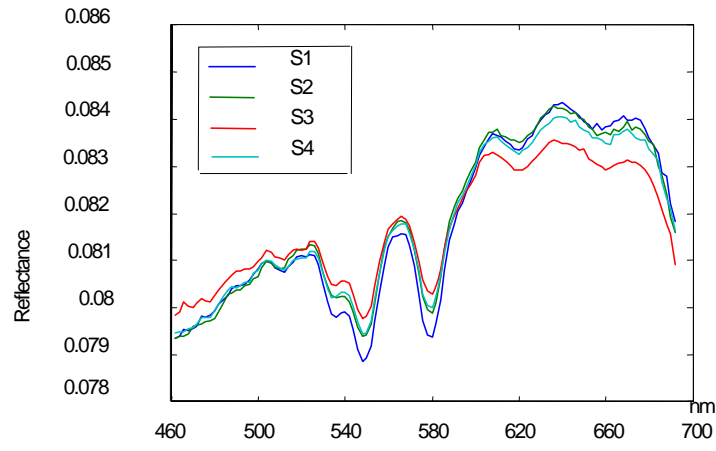

(a)

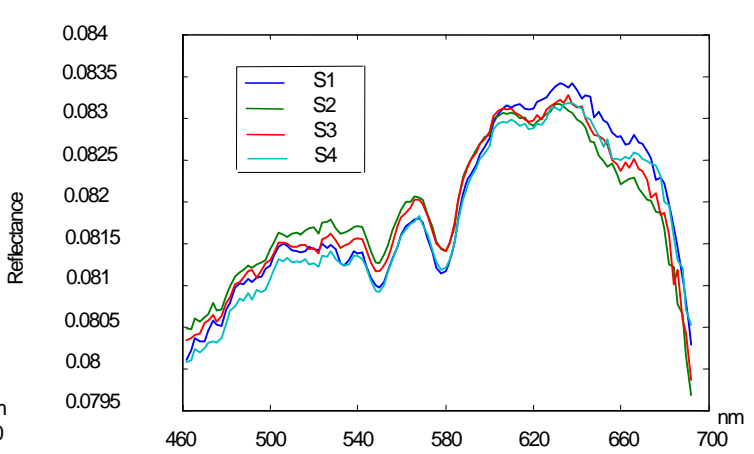

(b)

Fig. 4 Skin reflection of 4 individuals under two illumination conditions acquired by our sensing system whereby (a) is with higher light intensity than (b). 


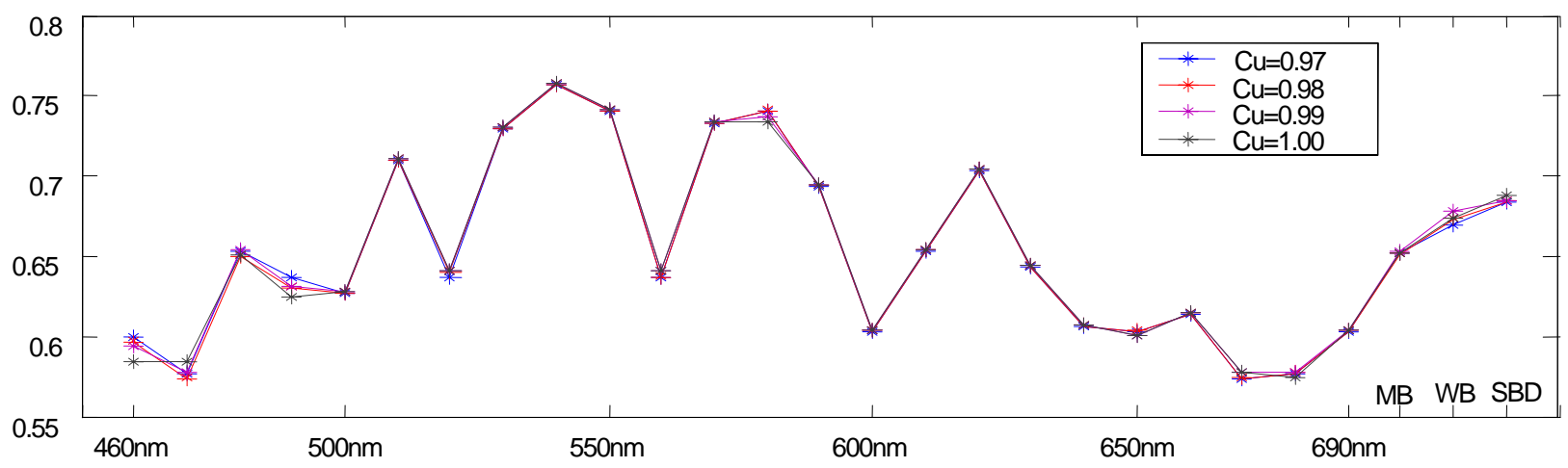

Fig. 5 Recognition rate of each single band by $(2 \mathrm{D})^{2} \mathrm{PCA}$ and by methods of $\mathrm{WB}(1,24)$ and $\operatorname{SBD}(24,1)$. 


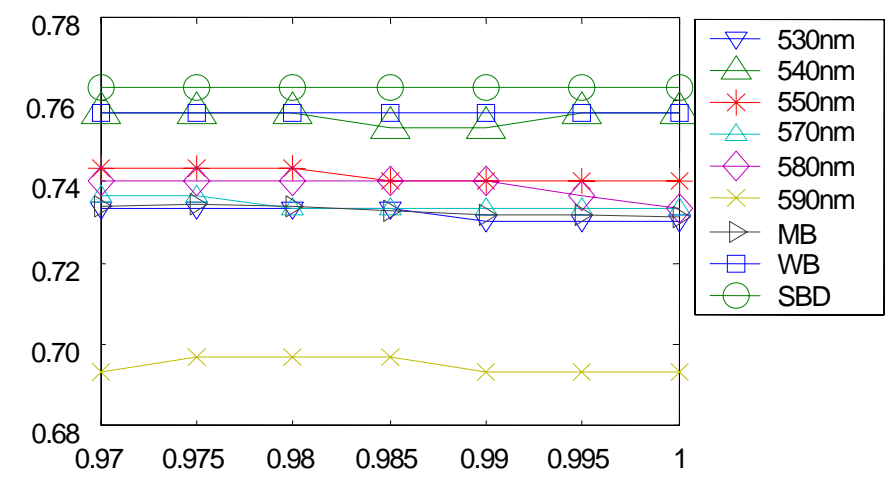

Fig. 6 Recognition rate of each selected single feature band and by methods of $\mathrm{WB}(2,3,3)$ and $\operatorname{SBD}(2,3,3)$. 


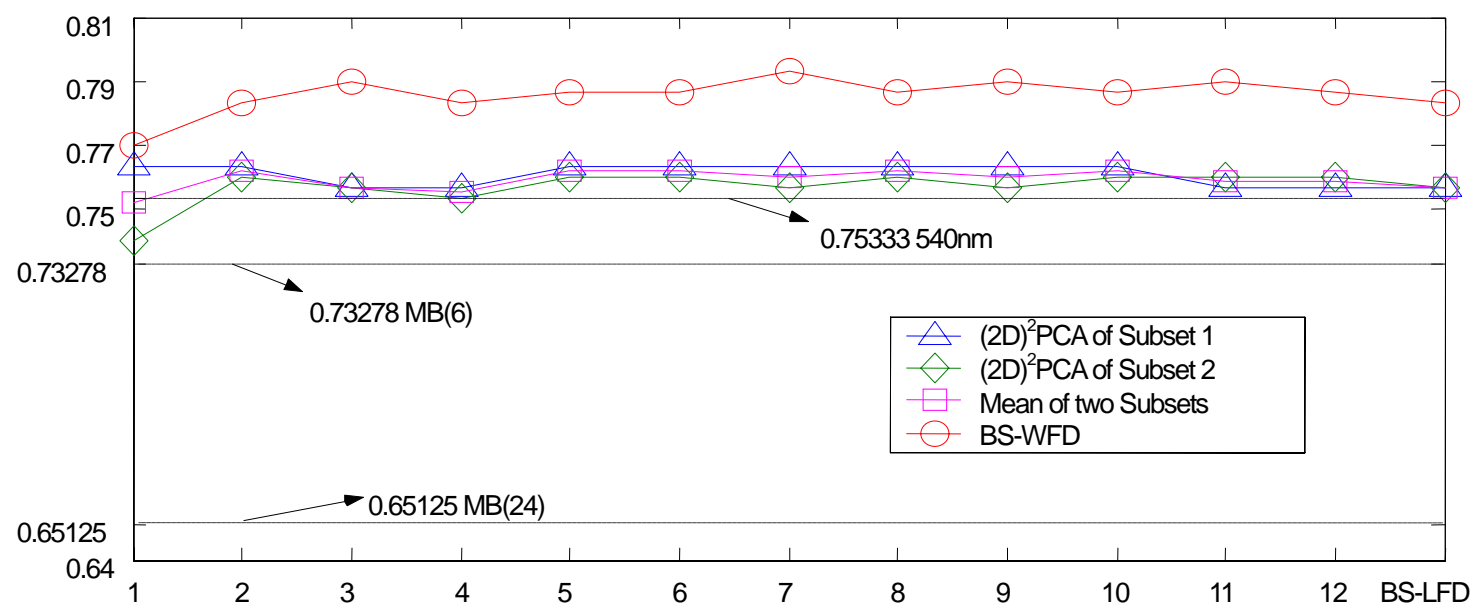

Fig. 7 Recognition rate of each subset by (2D) ${ }^{2} \mathrm{PCA}$, the proposed methods BS-WFD with 12 weighting cases (denoted from 1 to 12) and BS-LFD. 


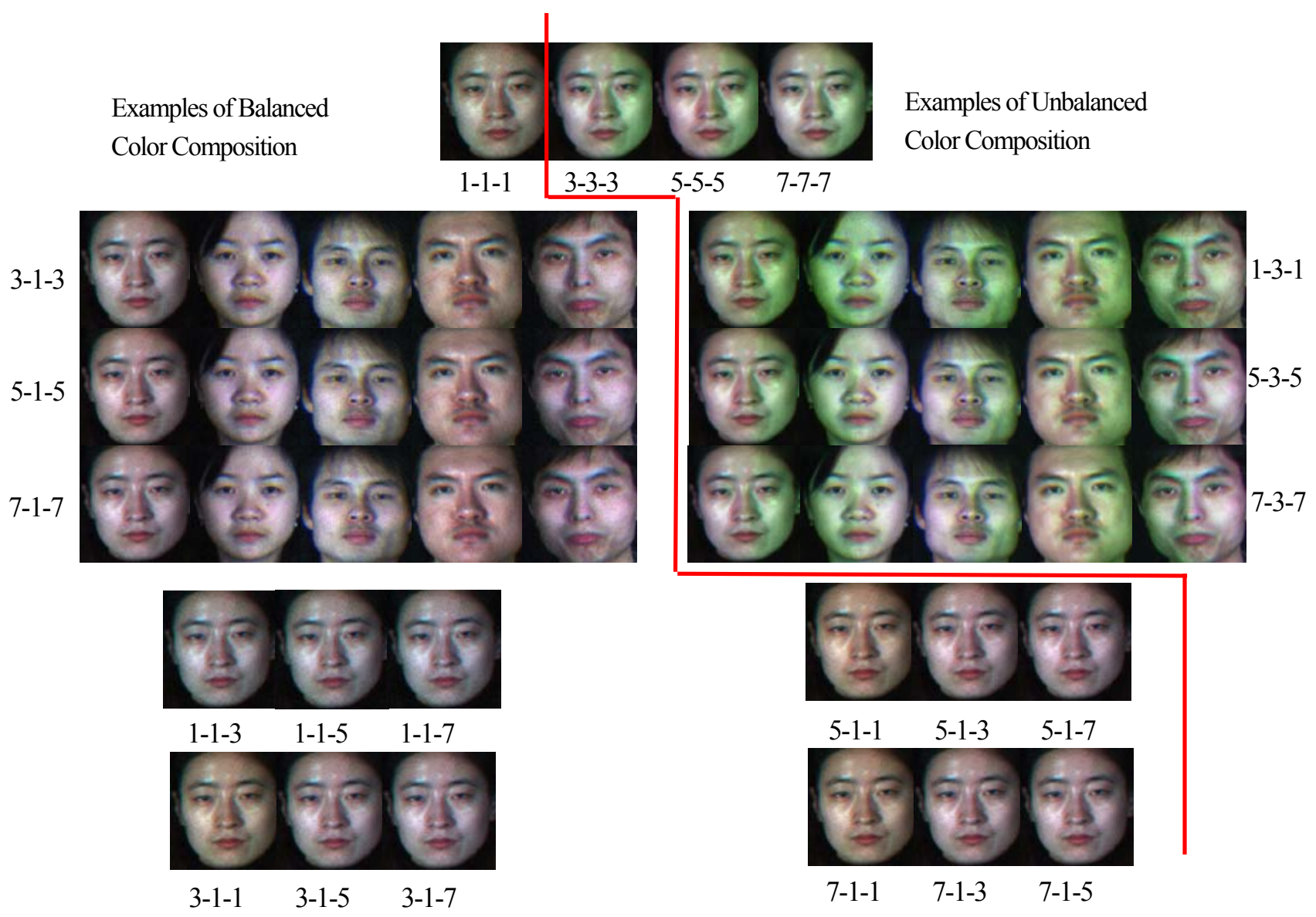

Fig. 8 Examples of RGB color image simulation. 


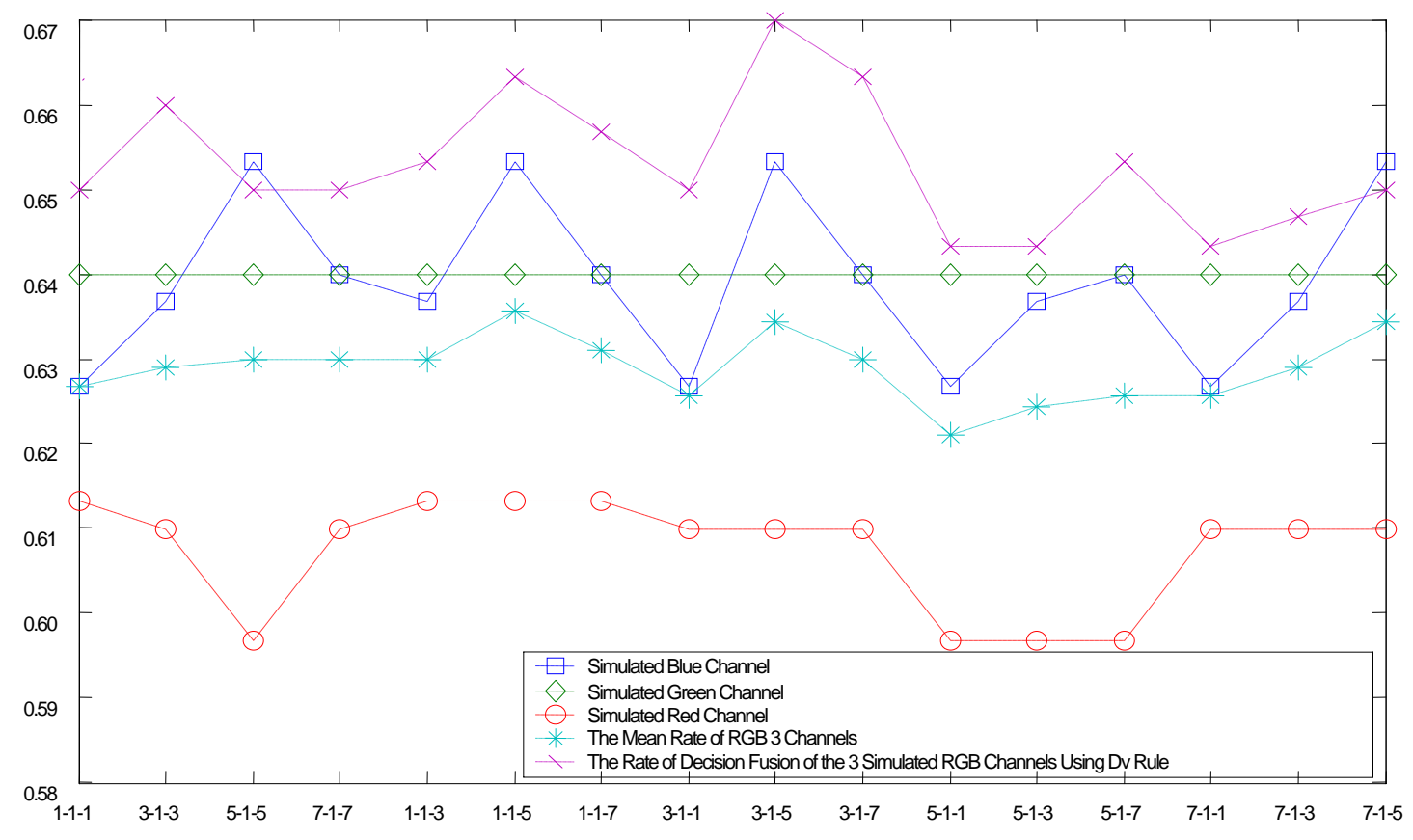

Fig. 9 Face recognition rate of simulated color images with balanced color composition. 


\section{List of Tables}

Table 1. A brief summarization of the recognition methods.

Table 2. Mean recognition rate of each single band by $(2 \mathrm{D})^{2} \mathrm{PCA}$ and by methods of $\mathrm{WB}(1,24)$ and $\operatorname{SBD}(24,1)$.

Table 3. Recognition rate of each selected single feature band and by methods of $\mathrm{WB}(2,3,3)$ and $\operatorname{SBD}(2,3,3)$

Table 4. Recognition rates of each subset by (2D) ${ }^{2} \mathrm{PCA}$, BS-WFD of 12 weighting strategies, and BS-LFD. ERD represents the Estimated Row Dimension and ECD represents the Estimated Column Dimension. 
Table 1. A brief summarization of the recognition methods.

\begin{tabular}{|l|c|c|c|l|}
\hline Name & Symbol & $\begin{array}{l}\text { Band } \\
\text { Selection }\end{array}$ & $\begin{array}{c}\text { Bands Used } \\
\text { (Total) }\end{array}$ & Recognition Method \\
\hline \multirow{2}{*}{ WB } & WB(1,24) & No & $24(24)$ & Eqs. (1)-(4); $N=1, I_{1}=24$ \\
\cline { 2 - 6 } & $\mathrm{WB}(2,3,3)$ & Yes & $6(24)$ & Eqs. (1)-(4); $N=2, I_{1}=3$ and $I_{2}=3$ \\
\hline \multirow{2}{*}{ SBD } & SBD(24,1) & No & $24(24)$ & Eqs. (1)-(4) and (6)-(8); $N=24, I_{i}=1,(i=1,2, \cdots, 24)$ \\
\cline { 2 - 6 } & SBD(2,3,3) & Yes & $6(24)$ & Eqs. (1)-(4) and (6)-(8); $N=2, I_{1}=3$ and $I_{2}=3$. \\
\hline \multirow{2}{*}{ BS-xFD } & BS-WFD & Yes & $6(24)$ & Eqs. (1)-(8) With varying weight assignment strategy \\
\cline { 2 - 6 } & BS-LFD & Yes & $6(24)$ & Eqs. (1)-(8) Laplacian Pyramid with salient measurement selection rule \\
\hline
\end{tabular}


Table 2. Mean recognition rate of each single band by $(2 \mathrm{D})^{2} \mathrm{PCA}$ and by methods of WB(1,24) and $\mathrm{SBD}(24,1)$.

\begin{tabular}{|c|c|c|c|c|}
\hline $\mathbf{C u}$ & $\mathbf{0 . 9 7}$ & $\mathbf{0 . 9 8}$ & $\mathbf{0 . 9 9}$ & $\mathbf{1 . 0 0}$ \\
\hline MB(24) & 0.65208 & 0.65152 & 0.65167 & 0.65082 \\
\hline WB(1,24) & 0.67000 & 0.67333 & 0.67667 & 0.67333 \\
\hline SBD(24,1) & 0.68333 & 0.68333 & 0.68333 & 0.68667 \\
\hline
\end{tabular}


Table 3. Recognition rate of each selected single feature band and by methods of $\mathrm{WB}(2,3,3)$ and $\operatorname{SBD}(2,3,3)$

\begin{tabular}{|c|c|c|c|c|c|c|c|}
\hline $\mathbf{C u}$ & $\mathbf{0 . 9 7 0}$ & $\mathbf{0 . 9 7 5}$ & $\mathbf{0 . 9 8 0}$ & $\mathbf{0 . 9 8 5}$ & $\mathbf{0 . 9 9 0}$ & $\mathbf{0 . 9 9 5}$ & $\mathbf{1}$ \\
\hline $\mathbf{5 3 0 n m}$ & 0.73333 & 0.73333 & 0.73333 & 0.73333 & 0.73000 & 0.73000 & 0.73000 \\
\hline $\mathbf{5 4 0 n m}$ & 0.75667 & 0.75667 & 0.75667 & 0.75333 & 0.75333 & 0.75667 & 0.75667 \\
\hline $\mathbf{5 5 0 n m}$ & 0.74333 & 0.74333 & 0.74333 & 0.74000 & 0.74000 & 0.74000 & 0.74000 \\
\hline $\mathbf{5 7 0 n m}$ & 0.73667 & 0.73667 & 0.73333 & 0.73333 & 0.73333 & 0.73333 & 0.73333 \\
\hline $\mathbf{5 8 0 n m}$ & 0.74000 & 0.74000 & 0.74000 & 0.74000 & 0.74000 & 0.73667 & 0.73333 \\
\hline $\mathbf{5 9 0 n m}$ & 0.69333 & 0.69667 & 0.69667 & 0.69667 & 0.69333 & 0.69333 & 0.69333 \\
\hline $\mathbf{M B ( 6 )}$ & 0.73390 & 0.73444 & 0.73389 & 0.73278 & 0.73167 & 0.73167 & 0.73111 \\
\hline $\mathbf{W B}(\mathbf{2 , 3 , 3 )}$ & 0.75670 & 0.75670 & 0.75670 & 0.75670 & 0.75670 & 0.75667 & 0.75667 \\
\hline $\mathbf{S B D ( 2 , 3 , 3 )}$ & 0.76333 & 0.76333 & 0.76333 & 0.76333 & 0.76333 & 0.76333 & 0.76333 \\
\hline
\end{tabular}


Table 4. Recognition rates of each subset by (2D)²PCA, BS-WFD of 12 weighting strategies, and BS-LFD. ERD represents the Estimated Row Dimension and ECD represents the Estimated Column Dimension.

\begin{tabular}{|c|c|c|c|c|c|c|c|}
\hline \multicolumn{2}{|c|}{ Weighted Fusion } & Subset 1 & Subset 2 & $\begin{array}{l}\text { Mean of two } \\
\text { Subsets }\end{array}$ & $\begin{array}{l}\text { Dv Combine } \\
\text { of } 2 \text { Subsets }\end{array}$ & ERD & ECD \\
\hline \multirow{12}{*}{ 屋 } & $1(1-1-1 \& 1-1-1)$ & 0.763333 & 0.740000 & 0.751667 & 0.770000 & 32 & 32 \\
\hline & $2(1-5-1 \& 1-5-1)$ & 0.763333 & 0.760000 & 0.761667 & 0.783333 & 33 & 34 \\
\hline & $3(1-10-1 \& 1-10-1)$ & 0.756667 & 0.756667 & 0.756667 & 0.790000 & 34 & 36 \\
\hline & $4(1-15-1 \& 1-15-1)$ & 0.756667 & 0.753333 & 0.755000 & 0.783333 & 35 & 37 \\
\hline & $5(3-10-1 \& 3-10-1)$ & 0.763333 & 0.760000 & 0.761667 & 0.786667 & 33 & 34 \\
\hline & $6(5-10-1 \& 5-10-1)$ & 0.763333 & 0.760000 & 0.761667 & 0.786667 & 32 & 34 \\
\hline & 7 (5-10-1\&1-10-1) & 0.763333 & 0.756667 & 0.760000 & 0.793333 & 33 & 36 \\
\hline & $8(5-10-1 \& 3-10-1)$ & 0.763333 & 0.760000 & 0.761667 & 0.786667 & 32 & 34 \\
\hline & $9(3-10-1 \& 1-10-1)$ & 0.763333 & 0.756667 & 0.760000 & 0.790000 & 33 & 36 \\
\hline & $10(3-10-1 \& 5-10-1)$ & 0.763333 & 0.760000 & 0.761667 & 0.786667 & 33 & 34 \\
\hline & $11(1-10-1 \& 3-10-1)$ & 0.756667 & 0.760000 & 0.758333 & 0.790000 & 34 & 35 \\
\hline & $12(1-10-1 \& 5-10-1)$ & 0.756667 & 0.760000 & 0.758333 & 0.786667 & 34 & 34 \\
\hline & BS-LFD & 0.756667 & 0.756667 & 0.756667 & 0.783333 & 41 & 41 \\
\hline
\end{tabular}

